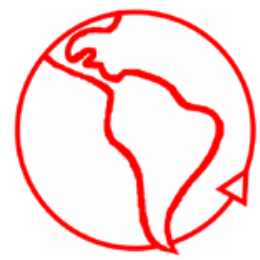

\title{
Dificultades y paradojas de la observación de segundo orden: Reflexiones en torno al cálculo de la forma
}

Difficulties and paradoxes of second-order observation. Reflections on the calculus of form

\section{Fernando Robles Salgado}

Departamento de Sociología y Antropología, Universidad de Concepción, Chile

\section{Resumen}

Este artículo enfoca las particularidades de la observación de segundo orden desde el punto de vista del cálculo de la forma, ideado por George SpencerBrown. En un primer paso, se destacan las posibilidades que para las ciencias sociales se abren con el uso de la forma como estrategia de observación. En un segundo paso, se establecen once tesis acerca de la puesta en uso de la observación de segundo orden. Específicamente, se redefine la teoría de la observación de segundo orden desde la teoría de sistemas de Niklas Luhmann.

Palabras Clave: Teoría de Sistemas; Observación de Segundo orden; Cálculo de la Forma; Paradojas de la Observación; Sistemas Sociales

\begin{abstract}
This paper focuses on the particularities of the second-order observation from the point of view of the calculus of form devised by George Spencer-Brown. In a first step, it highlights the potential opening up for the social sciences with the use of the form as observation strategy. In a second step, it provides eleven theses on the practical use of the second-order observation. Specifically, it redefines the theory of second-order observation from systems theory of Niklas Luhmann.
\end{abstract}

Keywords: Social Systems Theory; Second Order Observation; Calculus of Form; Paradoxes of the Observation; Social Systems

Introducción

"iTraza una distincióni"

George Spencer-Brown

Las reflexiones contenidas en la obra de Spencer-Brown 'Laws of Forms' (1997)' constituyen uno de los fundamentos de la teoría de la sociedad de Niklas Luhmann, y dan lugar al despliegue de una metodología independiente de acceso al mundo empírico desde la teoría moderna de sistemas. Durante su vida y principalmente en su obra principal 'Die Gesellschaft der Gesellschaft' (1997), Luhmann tematizó in extenso el problema de la observación en general y de la observación de segundo orden en particular: la introducción del observador en la construcción todo conocimiento del mundo, el fenómeno operacional de la observación y el epifenómeno de la observación de observadores. Además, en 'La ciencia de la sociedad' (1996) y en

\footnotetext{
${ }^{1}$ Citado en lo sucesivo según la traducción al alemán de 1997.
} 
'El arte de la sociedad' (2005), abordó en detalle dicha problemática referida no exclusivamente a la producción científica y artística. Numerosos artículos dedicados al tema ilustran su dedicación e interés sobre el tema.

1. El cálculo de la forma (CF) y la puesta en uso de las distinciones

Las leyes de la forma no son descripciones, sino comandos y órdenes. Spencer-Brown (1997) muestra que el famoso misterio formulado por Wittgenstein en el sentido de que el mundo que conocemos está construido de tal manera que es capaz de verse a sí mismo y que "las reglas de la sintaxis lógica deben ser inteligibles por sí mismas con sólo conocer cómo cada signo designa" (Wittgenstein 1957: 61-62), no es tal, sino que es algo que simplemente no podemos evitar. Esta constatación que parece abstracta e irrelevante, tendrá consecuencias decisivas para la construcción del conocimiento.

La primera instrucción operativa de las leyes de la forma ordena: 'Traza una distinción', lo que en realidad no tendría mayor trascendencia, sin la clave del operador multifuncional, antes indicado.

Fig. 1. Marca

Esta marca un signo del trazado de una distinción, tal como donde no hay nada que pueda distinguirse de nada, o como si en una hoja de papel en blanco, alguien dibujara un círculo, o lo que fuera. El operador señala que con el trazado de la distinción se efectuó una operación doble y recursiva: 'indicación/distinción'. Cuando se distingue se indica y cuando se indica se distingue. La asimetría entre la parte cóncava interior del operador y la parte exterior, es la que señala la posibilidad de la forma. Pero antes de la orden de trazar una distinción, o de marcar algo como una 'fractura' donde antes no existía nada, Spencer-Brown entrega 'dos definiciones' y 'dos axiomas' preparatorios.

- Las 'definiciones' nos dicen:

a) Una vez que se ha trazado la distinción, se pueden designar y marcar los espacios, los estados o los contenidos en cada uno de los lados de la línea divisoria;

b) No puede haber distinciones sin motivos y que no puede haber ningún motivo sin que los contenidos de cada una de las partes se hayan designado como diferentes en sus valores. Luego, cuando un contenido tiene un valor, se puede escoger un nombre para designar ese valor. Por lo que lo nombrado con el nombre, se puede identificar con el valor del contenido.

- Los 'axiomas' son los siguientes:

a) "Axiom I. The law of calling. The value of a call made again is the value of the call. That is to say, if a name is called and then is called again, the value indicated by the two calls taken together is the value indicated by one of them. That is to say, for any name, to recall is to call." (Spencer-Brown 1997: 2) La notación del primer axioma es designada como la forma de la 'condensación': 
Fig. 2. Axioma I

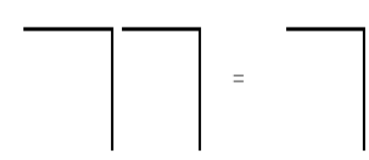

b) "Axiom II. The call of crossing. The value of crossing made again is not the value of the crossing. That is to say, if it is intended to cross a boundary and then it is intended to cross it again, the value indicated by the two intentions taken together is the value indicated by none of them. That is to say, for any boundary, to recross is not the cross." (Spencer-Brown 1997: 2) La notación del segundo axioma es designada como la forma de la 'cancelación'.

Fig. 3. Axioma II

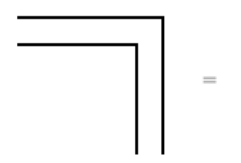

Previamente, queremos sintetizar algunas consecuencias de lo anterior. La forma para Spencer-Brown es una 'forma de dos partes'. Se trata de una distinción que tiene una parte interna y una externa, que se encarga de expresar el operador multifuncional.

La forma es el resultado de una operación que corresponde al axioma II, o del cruce desde la parte exterior de la distinción, el 'unmarked state', a la parte interior, el 'marked state'. Por lo tanto, la forma es una 'asimetría' o mejor dicho está construida sobre un fundamento asimétrico. La forma es el resultado de una operación, esta operación permite dos conexiones: una que es la 'confirmación' del lado marcado, la forma de la 'condensación', y con la segunda la 'cancelación', que porque regresa al lado no marcado, se elimina la distinción.

Ahora bien, en la concepción del cálculo de la forma, se introducen varios cánones.

- El primero se refiere a la función de la señal, es decir del indicador. La función es un acuerdo que recuerda que "lo que no está permitido, está prohibido." (Spencer-Brown 1997: 3)

- El segundo canon permite 'contraer las referencias'.

- El tercero, que se refiere a los acuerdos de sustitución, define con el signo ( $(\boldsymbol{)}$ la dirección del cambio, o también 'paso'.

- Con el quinto canon, Spencer-Brown describe las cuatro operaciones del cálculo de la forma. Como el 'cálculo', se ordena denominar un procedimiento con el cual, mediante pasos, una forma se transforma en otra y como 'un' cálculo, a un "sistema de construcciones y acuerdos" que permiten la calculación (Spencer-Brown 1997: 9-10).

Cada una de las operaciones tiene una operación inversa. De la forma de la distinción se obtienen la 'condensación' y la 'cancelación'; junto a sus operaciones inversas, hacen que se pueda expandir la referencia.

- La operación inversa de la condensación es la 'confirmación', o sea la ratificación de un crossing mediante otro que le sigue. 
- La operación inversa de la cancelación es la 'compensación', es decir, la introducción de una distinción que se cancele, en lugar del unmarked state.

Las operaciones de condensación y confirmación son operaciones numéricas, mientras que la cancelación y la compensación son operaciones de orden. Por ejemplo, las dos operaciones del primer axioma (law of calling), son numéricas, y las del segundo axioma (law of crossing) son de orden.

Únicamente con estas cuatro formas, Spencer-Brown construye su cálculo y puede expandirlo de diversas maneras, construyendo acuerdos de formas, que siempre pueden ser reducidos a la situación original de partida, al marked state y el unmarked state, los dos lados de la distinción. Deliberadamente, excluimos aquí la mayor parte de las consecuencias que se derivan del cálculo de la forma para las matemáticas y el álgebra (Simon 1993: 38 y ss.). Pero conviene recordar que el Cálculo de le Forma (CF), no es una simple reconstrucción; lo que realiza exitosamente Spencer-Brown es evadir la prohibición de autoreferencialidad formulada por Wihthead y Russell en 1927. Como es sabido, ya en 1931 Kurt Gödel (1940) había cuestionado el fundamento del álgebra tradicional. Spencer-Brown se apoyará en buena parte en dicho trabajo.

De entre los acuerdos más comentados del CF, se destaca el paso de los acuerdos finitos a los acuerdos infinitos. Esto lo consigue Spencer-Brown con la observación de la figura de la re-entry (Spencer-Brown 1997: 60) Esta figura se sustenta en la autosimilitud de una expresión infinita, o sea en la similitud entre una parte de una expresión con toda la expresión. Por lo tanto, re-entry significa la indicación de la distinción misma: dicho de otra manera, la observación de la forma de la distinción en el lado interno de la distinción. De especial importancia son las palabras finales del capítulo respectivo: "El valor de la dimensión de una circunferencia con relación al espacio externo debe ser el valor de la marca, porque la marca sólo distingue ese espacio. Un observador es del mismo modo una marca, porque distingue el espacio que ocupa”. (Spencer-Brown 1997: 65-66)

Fig. 4. Re-entry

Esta relativización radical del observador hasta el 'extremo de la invisibilidad' es de gran relevancia para la construcción de cualquier teoría de la observación (Winter 1999). "La observación es la que determina lo observado y no a la inversa, pero sólo se puede observar estando-en-el-espacio-mundo, para ser sólo una marca e idéntico a la forma" (Von Foerster 1996: 89 y ss.). Una marca que se confunde con el espacio, significa que el observador "no ve que no ve", es decir, que su observación tiene un "punto ciego" (Maturana \& Varela 1984: 6), que corresponde exactamente a la juntura de la distinción que construye. Todo el cálculo de la forma está destinado a que se pueda leer formalmente el punto ciego de la observación del observador.

Pero con ello, el mismo observador es idéntico no sólo con la marca, sino también con la primera distinción; es decir, con la marcación de un lado como el marked state, y con la forma. Con la operación de re-entry, se introduce un nivel tal de indeterminabilidad, que compromete cualquier posibilidad de observaciones 'precisas', pero al mismo tiempo se establece en el CF una propiedad central. 
Si consideramos que la definición elemental de la forma es perferct continence y si the law of crossing señala: "The value of crossing made again is not the value of the crossing" (Spencer-Brown 1997: 2), entonces tienen que haber dos maneras de cruzar el límite de la distinción:

a) Si se cruza desde afuera hacia adentro, se traza la distinción (esta es una operación que podemos repetir de acuerdo a the law of calling, cuantas veces queramos), pero,

b) Si se cruza desde adentro hacia afuera, entonces la distinción se elimina. Por lo tanto, el concepto de la forma tiene sólo sentido, si se opera desde afuera hacia adentro y no a la inversa.

Nosotros construimos una existencia en la medida en que sus elementos son descompuestos en una identidad triádica. La existencia se extingue cuando se unifica. Cada connotación implica dualidad, no podemos reproducir una cosa, sin co-producir algo que no es, y cada dualidad implica triplicidad: lo que la cosa es incluye lo que no es, es el juntura del medio, entre lo que es y lo que no es. (Spencer-Brown 1997: xviii)

Ahora bien, todas las expresiones entrelazadas que se guían por los dos axiomasleyes (calling y crossing) pueden expandir referencias o simplificarlas (Spencer-Brown 1997: 9). Las simplificaciones se ejecutan con las formas de 'condensación' y 'cancelación', mientras que las expansiones siguen la fórmula de la 'confirmación' y 'compensación'. Si consideramos los argumentos anteriores, desde la observación sociológica, se desprenden una serie de consecuencias fundamentales.

1.) La primera de ellas es que el mundo permite tantas observaciones como distinciones sean posibles y que todas las descripciones del mundo obedecen al uso (nos demos cuenta o no) de distinciones. El mérito del CF de Spencer-Brown consiste en adiestrar al observador en el uso de distinciones como operaciones propias de su propio observar.

Los modos de expansión y compresión de referencias, que corresponden a operaciones específicas de distinción, configuran modos de 'reducción de complejidad' diferentes, pero no hay ningún criterio metasocial para definir a las simplificaciones como privilegiadas respecto de las expansiones o viceversa (Luhmann 1998: 48-50), todo es dependiente del observador. Además, con el uso de un solo operador (la marca), Spencer-Brown hace posible la contención de uno o dos argumentos contradictorios; no es necesario agregar otro en el caso de que un objeto sea negado o afirmado, pues en ambos casos se trata de una operación de observación, lo que es una negación para una lectura del 'paso', puede ser una afirmación para otra lectura del paso siguiente.

Lo más interesante de la law of crossing es, sin embargo, que la configuración de la forma corresponde a una externalidad. Esto significa que en el empleo de distinciones, se puede hacer uso de ellas sin percatarse de ello, o bien se pueden observar las distinciones con las que se opera -de eso tratan justamente las leyes de la forma. Pero en cualquier caso, Spencer-Brown deja en claro que el conocimiento que se obtiene mediante el uso de distinciones, es un entrelazamiento de procedimientos operativamente clausurados, completamente cerrados y que no tienen ninguna correspondencia con el entorno, análogamente al argumento de Wittgenstein en 3.333 del Tractactus: "Una función no puede ser su propio argumento porque el signo de la función contiene ya el prototipo de su propio argumento y no puede contenerse a sí mismo" (Wittgenstein 1957: 59). Por ello es que las distinciones son construcciones que no tienen ninguna correspondencia con 
un entorno que se le impute alguna realidad independiente (Luhmann 2005: 97 ss.; Luhmann 2001)

2.) Segundo, si cualquier 'observación' puede ser transformada, mediante una 'descripción' que el propio observador considere adecuada y correspondiente, en un 'argumento', habría que formular dos interrogantes.

a) ¿Es posible que el propio observador se percate que detrás de su argumento hay una descripción, que a su vez resulta de una observación y que la observación arranca de un manejo más o menos complejo de distinciones?

b) ¿Cómo pueden ser observadas las operaciones de observación?

La primera pregunta ha sido respondida de muchas maneras. Para Husserl, la cualidad fundamental de la existencia humana es "el a priori del mundo de la vida", "este mundo es el constante suelo de la validez, es una fuente siempre lista de autoevidencias, una fuente a la que recurrimos sin más ni más, bien en tanto que hombres prácticos, bien en tanto que científicos" (Husserl 1991: 128), por lo que los pobladores de cualquier mundo de la vida, no se pueden percatar de las operaciones de observación auto-contenidas en sus descripciones, sean estos legos o científicos, trátese de descripciones de objetos o de autodescripciones -como ha demostrado Lacan (1973: 171-239). Por ello es que Spencer- Brown argumenta que el observador es sólo una marca, idéntica a la distinción, nada menos y nada más.

La respuesta a la segunda pregunta, por su parte, es un tema complejo. Mientras tanto, se podría obedecer la instrucción de Spencer-Brown: "stay where you are", que corresponde la forma de la compensación (Spencer-Brown 1997: 12).

3.) Tercero, ¿cuál es la significación de la marca, o que el cálculo se realice con un solo operador ${ }_{7}$ ? Heinz von Foerster ha celebrado con especial ironía el ingenio que implica esta "mark $\rceil$ of distinction" (Von Foerster 1993: 9-11) y compara a las leyes de la forma con una máquina de afeitar eléctrica transistorizada marca "Spencer-Brown", la que sin esfuerzo acaba con dos mil años de maleza semántica imponente y obstinada. Pero el operador no es cualquier signo como los de la aritmética, sino que lleva implicada la posibilidad del cruce y la distinción entre la parte interior y la parte exterior y de poder indicarlas. El cruce del límite tiene dos y sólo dos consecuencias: la confirmación de la distinción o su eliminación. Las formas law of calling y law of crossing (condensación y cancelación), las denomina Spencer-Brown "ecuaciones primitivas". Por lo tanto, es el operador ${ }_{7}$, el que permite distinguir entre el lado interno (marked state) y el externo (unmarked state).

Consecuentemente, las instrucciones-base del cálculo son solamente dos: 1) ¡Cruza! expresada en la marca y 2) ;Quédate dónde estás! -expresada mediante el unmarked state (Baecker 1993; Esposito 1993). De tal manera que el operador sería, como señala Torres Nafarrate, "el sustento universal de toda construcción de formas". Además, la fascinación del operador no consiste sólo en su "soledad" -es decir en la paradoja de su unicidad y multiplicidad-, sino que además su enorme influencia en la teoría de la sociedad de Luhmann, se refleja en el impulso de búsqueda de 'una sola operación' que pueda considerarse como genuinamente social, una especie de forma fundacional, de la cual se puedan expandir más y más referencias. "Esta operación es para Luhmann la comunicación". (Torres Nafarrate 1999)

4.) Cuarto, el cálculo de la forma rompe definitivamente con el esquema sujeto/objeto. Por un lado, suprime las cualidades ontológicas y altamente paradójicas con las que se había investido hasta ahora al 'sujeto', por lo tanto el observador no es ningún sujeto que se distingue de un 'objeto', sino que la distinción 
sujeto/objeto es observada como una construcción más hecha por un observador, que maneja distinciones.

El observador es un 'sistema observador', tal como lo describe la cibernética de segundo orden, que distingue/indica mediante el trazado de distinciones que se observan como formas. Si la forma se obtiene mediante el cruce del límite de la distinción, entonces para ello se necesita tiempo, es decir, una distinción entre "antes y después" (Luhmann 1996: 64). Por otro lado, distinguir/indicar, es decir trazar una distinción implica, presupone, como señala Spencer-Brown, un motivo. Este motivo por el cual se traza esa distinción (y se excluye otra), corresponde exactamente al imperativo de selectividad de los sistemas sociales.

5.) Quinto, lo que produce una distinción o muchas interconectadas por operaciones de confirmación $(\eta>\eta\rceil)$ o condensación $(\eta\rceil, \eta)$ o interrumpidas por medios de cancelación o compensación, no corresponde necesariamente a la forma 'verdad/falsedad', sino que origina una multiplicidad de formas, cuyas distinciones, según Varela, revelan las capacidades cognitivas del "distinctor" (Gougen \& Varela 1979). La forma verdad es una construcción que a juicio de Von Foerster constituye una especie de adaequatio intellectus et rei, el camaleón de la filosofía, que según el usuario va cambiando de color (Von Foerster 1998: 29)

6.) Sexto, nosotros pensamos que en las definiciones y los axiomas de George Spencer-Brown previos a la instrucción: 'Traza una distinción', se formula un principio de abstención explícito. En efecto ¿por qué la instrucción básica se formula recién en el capítulo 2 del libro? Aquí no se trata de modificar esta exigencia por otra precedente, sino de dar cuenta de la propiedad elemental de la distinción, a saber: "Distinction is perfect continence" (Spencer-Brown 1997: 1), que leemos en el capítulo 1. Si la distinción es continencia perfecta y recién en el capítulo 2 bajo el título "Construcción", aparece la instrucción "Traza una distinción”, entonces lo que significa que la distinción sea continencia perfecta, "that is to say, a distinction is drawn by arranging a boundary with separstes sides so that a point on one side cannot reach the other side without crossing the boundary" (Spencer-Brown 1997: 1), es sólo una definición previa, de la que desprende la enunciación de las dos leyes: "the law of calling" $y$ "the law of crossing." (Spencer-Brown 1997: 2)

Pero todavía no se ha trazado ninguna distinción. Nosotros entendemos todo el contenido del capítulo 1 como una serie de 'pasos instructivos preparatorios' al trazado de la distinción. Con un poco de imaginación, el capítulo 1 puede leerse como No traces (todavía) ninguna distinción. Por ello es que Dirk Baecker sostiene que "la abstención total de la distinción es una construcción, que precede a todo lo posible y que por lo tanto se dirige a poder observar 'todo lo posible"' (Baecker 1993: 19). Pero ¿qué significa poder observar 'todo lo posible’?. ¿Cuál es la significación práctica de esta operación de abstención e inhibición, si consideramos que la observación es una operación social, comunicativa, empírica y práctica? En el sentido de Von Foerster, esta es una "pregunta legítima", porque no tiene una respuesta definitiva, o bien puede tener varias respuestas. (Von Foerster 1990)

Nosotros quisiéramos ensayar una posible respuesta. En el tercer acápite de la definición de la distinción, en el capítulo 1, ("Distinction is perfect continence"), leemos lo siguiente: "Sin motivo no puede haber ninguna distinción y no puede haber ningún motivo sin que los contenidos sean vistos como diferentes en valor" (SpencerBrown 1997: 1) ¿Qué contenidos y qué valor? Respuesta: "Si se ha trazado una distinción, pueden ser designados los espacios, los estados o los contenidos en cada una de las partes del límite, en la medida en que sean diferentes". (Spencer-Brown 1997: 1). Pero nunca antes. De las reflexiones anteriores se puede extraer una 
conclusión simple y al mismo tiempo inaudita: Mientras no haya nada sino abstinencia, desmotivación, desinterés e indiferencia -todas propiedades que se obtienen de la conservación registrada de acontecimientos- se realiza en la práctica la instrucción 'No traces (todavía) ninguna distinción'. Este sistema en 'estado de espera' es, tal como Luhmann describe a los sistemas sociales en doble contingencia, una condición para la observación de las distinciones (Luhmann 1998: 113 y ss.)

La operación de re-entrada (re-entry) de la forma en la forma, si termina en una cancelación, $7 \mathbf{\sim}$ la distinción se simplifica por eliminación, pero si sigue la forma de una compensación $\rightarrow$ se expande. No obstante, la operación de re-entry que SpencerBrown describe mediante cuatro experimentos realizados usando círculos en lugar del operador, nos dice que a pesar de que el concepto de la forma consiste en la demanda de distinguir, aún "suponiendo esa exigencia, no podemos escapar de la forma, a pesar de que la podemos ver del modo que nos guste" (Spencer-Brown 1997: 60)

Por ello es que el resultado de la operación de re-entry es aparentemente insólito: la primera distinción (el círculo trazado en un espacio vacío), la marca y el observador no sólo son intercambiables (=), sino en la forma idénticos. En el comentario del capítulo 12 al que nos referimos, se describe mediante tres ejemplos, que cualquier forma es completamente dependiente de la observación, y el hecho de que la distinción y la forma sea el fundamento de la observación, es precisamente lo que convierte en un misterio que el mundo descubra sus capacidades de verse a sí mismo. "Frente a la forma, en la que nosotros mismos aceptamos que existimos, el misterio aparece probablemente de nuestra perseverancia por formular una pregunta, donde en realidad no hay nada que preguntar" (Spencer-Brown 1997: 91). De acuerdo a las leyes de la forma, donde aparezca esa capacidad del mundo, el estado y la condición que de ello resulte, son absolutamente inevitables, por lo que en realidad no hay ningún misterio. Como actores universales, podemos registrar la ley universal tan ampliamente como para decir: etcétera, etcétera, construirás el universo con cada detalle y potencialidad, tal como lo has hecho hasta ahora (Spencer-Brown 1997: 92).

Nosotros formulamos la siguiente pregunta: ¿Si el observador es la marca, donde está entonces el límite (marca) de la forma? Desde una lectura matemático-cognitiva de las leyes de la forma, Fritz Simon formula la siguiente respuesta: "El límite que se configura mediante la piel del observador, construye la distinción dentro-fuera" (Simon 1993: 56). Si marca la parte exterior de la distinción, su entorno, el mismo se convierte en el lado no marcado y construye junto al lado marcado el espacio, el estado y el contenido del mundo, pero -recalcando lo que aquí hemos sostenido- si marca el lado marcado, será retratado únicamente como una parte de la forma completa del mundo.

Una segunda consecuencia apunta a la dependencia contextual de las distinciones. Trátese del operador o de un círculo, cada uno de ellos por separado no es todavía ninguna distinción, por lo que no puede existir algo como la forma del círculo o la forma de la marca. Esto significa que un círculo o el operador no tienen forma sin su contexto implícito, por ello es que la instrucción: ¡Marca!, no puede ser confundida con que el círculo o el operador ya sean distinciones 'en sí', porque así fácilmente estaríamos reeditando la antigua distinción entre materia y forma. Probablemente por ello es que Spencer-Brown señala que el comando "Traza una distinción", puede expresarse también como:

- deja ahí una distinción

- encuentra una distinción 
reconoce una distinción

- describe una distinción

- define una distinción o,

- deja trazar una distinción (Spencer-Brown 1997: 73)

Si observamos con un poco de imaginación las traducciones que el propio SpencerBrown ofrece para la instrucción activa elemental de la distinción, es decir trazarla, podemos postular que ninguna de ellas puede realizarse extra-contextualmente.

- En efecto, la instrucción 'deja ahí una distinción' es equivalente a 'quédate dónde estás', o 'no traces (todavía) ninguna distinción propia', tal como hemos venido sosteniendo con el principio de abstinencia en la observación de segundo orden.

- 'Encuentra una distinción', significa que no hay ni puede haber ninguna distinción 'a priori' y que tal como hay distinciones que se pueden dejar como están, hay otras que hay que buscar y encontrar. El observador de segundo orden se propone precisamente encontrar una distinción donde hay opacidad, latencia, caos y sólo observaciones de primer orden.

- 'Reconoce una distinción', quiere decir que hay distinciones que trazamos sin poder ni querer reconocerlas como tales, o porque no nos interesa $o$ simplemente debido a que nadie en su sano juicio se pone a escarbar, tras verter una expresión en una conversación cualquiera, cuál fue la distinción que usó.

- 'Describe una distinción' tiene que ser necesariamente el resultado de una observación, de la que arranque la descripción, porque no se puede describir nada que no se haya observado, de la manera que sea. No obstante, quien describe distinciones puede abarcar sólo el lado marcado, con lo cual la distinción no se distingue (explícitamente) de nada, o procurar marcar los dos lados de una distinción ajena mediante el intrincado procedimiento que hemos venido describiendo. 'Dejar trazar una distinción' es lo realmente novedoso de todas estas equivalencias. Esta paridad significa que con la distinción que antes trazamos entre 'distinción propia' y 'distinción ajena' -lo que no es muy original pues se deriva fácilmente del concepto de comprensión ajena [Fremdverstehen] de la teoría de la comprensión- no solamente apuntábamos a una cuestión de procedimiento. A nuestro entender, si dejamos únicamente que se tracen las distinciones y no las podemos ni queremos observar, entonces cualquier teoría de la observación perdería completamente su razón de existencia.

En la comunicación de la sociedad, y en particular en los sistemas sociales, pero además cuando el observador se propone la observación de sistemas funcionales parciales de la sociedad, no se puede reconocer, definir, encontrar ni describir ninguna distinción ajena sin acceder a la forma específica que asume y demuestra asumir el contexto en uso de quienes las producen, comunican y hablan/conversan. Dicho más claramente aún: todas las distinciones ajenas, y obviamente también las de la observación de segundo orden, por estar acopladas a contextos, deben ser indexicales. (Robles 2002; Fontdevila, Opazo \& White 2011; Eckert 2008)

Que el CF haya sido privilegiado por un pensador tan excepcional y atrevido como Luhmann (Luhmann 1998), debe darnos que pensar. Tan flexible es el CF que ha inspirado artículos como 'La forma de la Universidad' (Stichweh 1993a), 'La temprana forma de la moneda' (Hutter 1993a), 'El otro lado de la enfermedad' (Simon 1993a), 'EI lado oscuro de la carrera' (Corsi 1993a) y otros tantos recopilados por Dirk Baecker (1993a) 
Elena Esposito (1993) ha logrado demostrar que con el aparato policontextural y el $\mathrm{CF}$, se puede obtener mucho más que lo que uno pueda imaginarse. En efecto, la figura de la re-entry, que aparece en los capítulos 11 y 12 del libro 'Laws of Form' (Spencer-Brown 1997), es decir, cuando el CF ya se ha descrito suficientemente, establece un grado de indeterminabilidad incalculable, tanto que la posibilidad de observaciones diferentes se convierte en completamente imprecisa e indefinida. Ya no es posible remitirse a la aritmética, sino que debieran definirse nuevas reglas de procedimiento, cuestión que Spencer-Brown deja en suspenso (Esposito 1993: 98).

Spencer-Brown se da por satisfecho con una curiosa forma de desmitificación, argumentando que no hay ningún misterio en ellas, por lo cual no son interesantes. Lo contrario es verdad, y eso convierte, con la re-entry, al CF precisamente en atractivo. Gotthard Günther sostiene que cualquier cálculo que implique un nivel superior de observación, debe ser 'polivalente'. EI CF de Spencer-Brown, al contrario, es estrictamente 'bivalente'. Las distinciones tienen únicamente dos lados y la bivalencia de la aritmética y del álgebra no se cuestiona en absoluto. ¿Debiera entonces ser desechado, como argumenta Kaehr (1993)? Para la lógica podrá no ser de interés, pero sí para el constructivismo².

En efecto: con la observación de observaciones se supone que se 'supera' la observación de primer orden. Pero ello, como antes hemos demostrado, no significa que no exista o que sea mermada en interés y relevancia. Si en la observación de primer orden se observan 'objetos', quien quiera observar deberá observar el modo en que ellos son observados. En estricto rigor, el comportamiento del observador de segundo orden no difiere del de primer orden. El problema está en los objetos noobservados, si se considera que la observación como tal es una operación restrictiva y discriminatoria. Suponer que los objetos pueden ser distintos a los que son, es decir, de una alta contingencia, nos obliga a hablar de 'objetos secundarios' no observados. La alta contingencia significa que junto a los 'objetos primarios' hay también 'objetos secundarios'. En esa bivalencia (unos al lado de los otros) se fundamenta la lógica de Günther. La multivalencia no consiste en que entre cero y uno haya algo probablemente entre medio, sino en la multiplicación de la bivalencias. Los observadores polivalentes se observan estrictamente separados de los observadores bivalentes (Günther 1976).

La relación con el mundo es la del observador de primer orden y su única lógica posible es la lógica bivalente. Si se opera al nivel de observación de orden más alto, la observación de primer orden aparece como la más pobre de condicionamientos. El significado fundamental del CF consiste el que ofrece una formalización para la observación de primer orden. La re-entry es únicamente el punto en que se explicita ese orden de observación. Pero dicha re-entry no sería posible sin la serie idiosincrásica de propiedades del CF. Dicha propiedades suponen la re-entry, por un lado: una observación de primer orden que renuncia a su estatus privilegiado, debe estar en condiciones de indicar lo que no ve. Por ello es que en el campo de una relación más amplia, su debilidad es su fortaleza. (Russell 1972).

\footnotetext{
${ }^{2}$ El CF ha sido criticado sobre todo desde la lógica policontextural del discípulo de Gothard Günther, Rodolf Kaehr (1993). Si confrontamos la claridad y sencillez del CF con el grado de abstracción, el lenguaje negativo (Hegel), la kenogramática y la teoría de los números cualitativos, todo esto sumado a una horripilante pretensión de racionalidad de Günther (2002) y su predilección por los esquemas incomprensibles, explicados en un lenguaje tan misterioso y contradictorio como el del mismo Hegel, entonces podemos entender que, "la ventaja del $\mathrm{Cl}$ (CF) frente a la kenogramática fue su sencillez, por eso fue de inmediato bien recibido e intensivamente desarrollado por el BCL...Ese motivo y el hecho que la kenogramática no fue considerada en la recepción alemana." (Kaehr 1993: 154-155)
} 
Otra de las particularidades del CF consiste en que usa un solo y único operador, con el cual se realizan todos los cálculos. Estos cálculos contienen siempre un operador de negación y vincula a muchas fórmulas entre sí. En Spencer-Brown un solo operador acepta uno o dos argumentos, en ambos casos se trata de operaciones de observación, en decir, la observación y la no observación contextualizada y aceptando además ese "contexto como implícito" (Varga \& Matzka 1993) En otras palabras, en el CF la negación no es el primer operador, porque de acuerdo al constructivismo primero hay que construir para poder negar. La negación presupone una operación básica: la cesura que lleva a un objeto respecto de otros se distinga y se indique, expresado en la marca, en la que se sustenta el cálculo de Spencer-Brown (Spencer-Brown 1997: 30).

La re-entry que se representa mediante la fórmula $a=f(a)$ corresponde al juicio que algo que no es lo que es (o es, lo que no es).Si la función $f$ no representa la igualdad entonces la fórmula muestra que $a$ es el resultado de una manipulación. Que esta reflexión no es el resultado de especulaciones abstractas, lo demuestra el caso de las operaciones binarias en un computador. Los computadores no presuponen la diferencia entre cero y uno y trabajan binariamente. Pero ellos no representan absolutamente nada, sólo hay operaciones. Todos los programas computacionales trabajan con la operación del 'paso siguiente' que posibilita que un algoritmo pueda pasar el otro siguiente. Con la fórmula siguiente: $\mathbf{i}=\mathbf{i}+1$. Se trata de una típica función de sí misma. El valor de la variable i es dependiente del valor de i. En todos los lenguajes computacionales primero se calcula la expresión del lado derecho de la ecuación y luego el resultado del lado izquierdo de la ecuación. La expresión del lado derecho se calcula antes que la orden se ejecute. El tiempo contribuye a la desparadojización. Los programas computaciones se basan implícitamente en una secuencia temporal de operaciones y sus lenguajes no permiten la asimetría entre verdadero y falso, de lo verdadero resulta sólo lo verdadero, mientras que de lo falso resulta cualquier cosa (Kelly-Bootle 1990). A Spencer-Brown le interesa sólo la articulación de operaciones, es decir la indicación de los estados que resultan de la primera distinción. La distinción es absolutamente simétrica y la negación incluida las posibles asimetrías es un operador derivado.

\section{Las propiedades elementales de la observación de segundo orden}

Nosotros no estamos interesados en destacar las formas de proceder de las teorías de la observación que hasta hoy se conocen, sino en desplegar esfuerzos en pro de una teoría de la observación que dé cuenta de las argumentaciones anteriores. Vale decir, que considere el cálculo de la forma de Spencer-Brown y la epistemología biológica de Maturana, de la cibernética de segundo orden de Heinz von Foerster y de la lógica policontextural de Gotthard Günther y que se entienda como una teoría acoplada a la teoría de los sistemas sociales, que tematiza Niklas Luhmann. A continuación presentamos un conjunto de 11 tesis relativas a esta superación de las teorías de la observación anteriores.

\subsection{La observación es una construcción del observador}

La primera reflexión que guía este propósito es la siguiente: observar no implica ningún acceso a una realidad ubicada en el 'exterior'. La distinción interior/exterior es una construcción, producida por un observador y equivale a la re-entry de la forma interior/exterior en el marked state interior. Esta constatación no sólo es válida para la distinción anterior, sino que confronta a cualquier observación con la siguiente paradoja: todo lo que se observe tiene que considerar que el observador ya estuvo presente. Por consiguiente, la observación no puede ser sino la construcción de un observador más. 
Si en el lugar de la internalidad/externalidad se colocan distinciones/indicaciones, es decir, la producción de la forma, se distingue lo que sucede de lo que no sucede. Si esta operación se va reiterando, se despliega un límite de un sistema que encierra lo que observa en sí mismo y con sus propios medios, análogamente a la configuración del "borde autónomo", una membrana que regula activamente el intercambio metabólico y que constituye la unidad física y la identidad del organismo (An der Heiden 1986). Por ello, el observador debe ser un sistema necesariamente clausurado y autopoietico. Dicho claramente, el sistema observa como observa y observa como puede. "Existe un número ilimitado de posibles formas de observación. Sin embargo, cuando varios observadores seleccionan una determinada distinción, operan en el mismo sentido" (Luhmann 2005: 97). Por lo tanto, no cualquier metodología cualitativa es una "observación de observadores", como insinúa Flores (2009)

\subsection{La observación es un manejo de distinciones contextualizadas}

Todo lo observado depende de la distinción que el observador utilice. Por lo tanto, se puede distinguir, en principio, cualquier 'cosa' de cualquier 'cosa'. Esta relatividad de la observación se entiende sólo como un 'manejo de distinciones', e incluye por supuesto a las operaciones clausuradas del sistema nervioso. Sin embargo, si consideramos que en el cálculo de la forma de Spencer-Brown, para que haya distinción hay que definir espacios y valores a cada uno de los lados de la distinción, el marked state se identificaría con el 'valor' ( $x$ ) y la magnitud asimétrica del unmarked state con el 'no-valor' (y), o por lo menos con algo distinto del 'valor marcado'. La asignación del valor es completamente arbitraria, como la letra $f$ de una función, que no significa nada (Wittgenstein 1957: 59). Como la observación directa e inmediata no se puede observar a sí misma, porque para eso tendría que poner en uso otra distinción, la distinción que produce la observación tampoco es objeto de la observación. Cuando se observa un objeto físico, no se observa la distinción entre observación y objeto, sino que al objeto como distinto de otro, o simplemente de cualquier otredad. La tesis afirma la existencia de contextos generales y particularizados entre la diferenciación funcional de la sociedad y la diferenciación particular de los sistemas de función, que tienen como propiedad elemental la reproducción autopoiética y la autoorganización en el nivel de la observación de segundo orden (Luhmann 2005:120). Todos estos contextos poseen propiedades indexicales (Garfinkel \& Sachs 1986)

\subsection{La observación es indeterminada, paradójica y arbitraria}

Las observaciones no pueden ser exactas, sino que se caracterizan por su 'arbitrariedad' e 'indeterminabilidad'. En principio, una observación no puede autoobservarse al tiempo que observa. Sin embargo, las observaciones son operativamente autónomas y la distinción es la base de la observación, porque de otro modo se observaría algo que sería 'otro'. La restricción de cualquier observación consiste entonces en que no se puede introducir una distinción de manera autoimplícita, porque la distinción es siempre una forma de dos lados ${ }^{3}$. No se puede distinguir sin antes haber distinguido. En otras palabras, no se puede distinguir sin indicar, las dos operaciones son simultáneas e interdependientes. A pesar de todo lo anterior, es posible introducir medidas que reduzcan la indeterminabilidad en la observación, metódicas restrictivas que reduzcan la inseguridad en medio de la contingencia generalizada (Luhmann 1992: 93-128). Pero sólo hasta cierto punto, pues las paradojas le permiten oscilar al observador brevemente entre comprobación

\footnotetext{
${ }^{3}$ Husserl ha demostrado que ni siquiera en las estructuras noésico-noemáticas de la "conciencia pura" es posible la exclusión de una distinción. (Véase Husserl, 1980: 194 y 197)
} 
y su contrario, pero no le permiten ir más allá, salvo que opere contra-intuitivamente (Luhmann 1990a: 128 ss.)

\subsection{La observación no persigue la verdad}

La observación no puede distinguir entre verdad y falsedad, sino que sólo opera como opera. Si la distinción es el fundamento de la observación, y la distinción no se observa a sí misma, entonces, esto significa que no ve que no ve. En otras palabras, la distinción es ciega respecto de sí misma, la observación pone en uso su propia distinción como su propio punto ciego, lo hace disponible sin ninguna necesidad de explicarlo. Por ello, todas las observaciones son ingenuas, o cándidas, en su nivel operativo. "Truth: the invention of a liar" (Von Foerster 2003)

Nosotros queremos reformular el tema de la posibilidad de la observación de observaciones o también llamada 'observación de segundo orden': ni la observación de una distinción se libra de la ingenuidad, trátese de la propia o de una ajena. Por ello es necesario remarcar que una distinción que opera independiente de la distinción operación/observación, que indica sólo que la autopoiesis de la comunicación de la sociedad funciona. Es decir, "la observación de segundo orden observa únicamente cómo se observa" (Luhmann 2005: 108)

Por ello distinguimos entre auto-observación u 'observación propia' y heteroobservación u 'observación ajena', lo que no se vincula a la distinción autoreferencia/heteroreferencia, porque ella está contenida en cada una de las distinciones, tanto en la propia como en la ajena. Por 'distinción propia' entendemos todas las operaciones de distinción e indicación que un observador efectúe independientemente de otros observadores; o el uso de valores propios para marcar uno o ambos lados de la distinción, sin que interesen las distinciones de otros observadores. (Luhmann 2005: 108)

Por 'distinción ajena', por su parte, entendemos todas las operaciones de distinción e indicación que efectúe un observador 'observando' las distinciones de observadores ajenos. La diferencia fundamental entre la distinción propia y la ajena es que la primera no se entrelaza con otras observaciones, o si lo hace, el observador no se percata de ello, mientras que la segunda se propone deliberadamente la observación de las distinciones que no son las suyas, pero para lo cual no puede dejar de operar con las suyas, aún cuando su observación se entrelace exitosamente con las observaciones ajenas. "La observación de segundo orden se distancia del mundo para llegar finalmente a omitir su unidad (integridad, totalidad) y abandonarse a lo que aparece como 'valor propio' en el proceso recursivo y dinámico de la observación de observaciones" (Luhmann 2005: 102).

Independientemente de la complejidad del entramado de distinciones de las observaciones, en el caso de arquitecturas de distinciones más complejas, la observación ajena como observación de la observación no conduce a conclusiones definitivas, como las de derivar de un dominio que produce una distinción, la propiedad generativa de un segundo que brota de improviso. (Maturana 1998:77). Además, hay que tener claro que no puede existir ninguna observación, cuya autoindicación no sea bloqueada por una paradoja; luego, la observación es una operación paradójica (Luhmann 1993: 212)

\subsection{Las propiedades del observador no se incluyen en la observación}

Las distinciones no dicen nada sobre las capacidades cognitivas de quien opera distinguiendo (Gouden \& Varela 1979). Si a pesar de la imposibilidad de exclusión del 
punto ciego y de la ingenuidad operativa, se pueden observar observadores, es decir, se pueden observar las distinciones con las que operó una observación, no es suficiente poner atención a las distinciones que usa. Por supuesto que quien se proponga observar distinciones debe usar distinciones propias y distintas de las que observa. Pero si así procede, ¿cómo lo hace para poder observar las ajenas?

La distinción entre observación de primer y segundo orden es hasta cierto punto, una ficción. Si el observador de segundo orden opera con distinciones diferentes a las de los observadores de primer orden, no tiene ninguna posibilidad de observarlas, por lo que, en primer lugar, tiene que abstenerse, inhibirse, guardarse, invisibilizarse, y apoyarse en la instrucción: 'No traces (todavía) ninguna distinción', después adquiere validez la recomendación de Luhmann: "o se participa en la observación o no se observa" (Luhmann 1996: 67). Las posiciones "neutrales" no existen, pero para que el sistema de observación sea efectivamente cerrado en sus operaciones, hay que saber esperar. ¿Qué es lo que tenemos que esperar?

La respuesta es muy simple: tenemos que esperar hasta tener valores-datosregistros, que nos permitan marcar como distintos ambos lados de la distinción que observamos. En la observación de segundo orden, es probable que esto no se consiga nunca, porque los datos-valores mutan rápidamente. Pero los valores $(x)$ del lado marcado los podemos obtener de lo que el observador de primer orden dijo/comunicó, la incógnita es la otredad, porque la magnitud del valor (y) del lado no marcado es desconocida y justamente allí se ubica el observador de segundo orden, por lo que el unmarked state de la observación de primer orden tiene que ser su propio marked state. Por ello es que la forma sólo tiene asidero en la observación de segundo orden. Además, no hay que caer en la equivocación fatal de analogizar los lados de la distinción con el gradiente de complejidad sistema/entorno, porque los dos lados de la forma son el sistema que observa.

Entretanto, antes del crossing, podemos perfectamente apoyarnos en la instrucción básica de Spencer-Brown: 'Quédate dónde estás'. En la observación de segundo orden, el observador no puede conformarse con ilusiones, con alimentar quimeras, por lo que está obligado a 'esperar'. $Y$ "se podrá hablar de observaciones de segundo orden cuando dos observaciones se acoplan de tal manera que satisfacen las características de una observación de primer orden" (Luhmann 2005: 106)

\subsection{El tiempo de la observación}

La observación opera sobre distinciones temporalizadas. En este sentido, hay que considerar que si hay una diferencia elemental entre la observación de primer y de segundo orden, es que la observación de segundo orden es siempre posterior, se retrasa, llega atrasada, es destemporalizada respecto de la observación de primer orden. Esta es la différence, la no-simultaneidad del presente consigo mismo, que tematiza Derrida (1972). Esta asincronía mundana es el recurso que la observación de segundo orden, se debe usar para reconstruir-deconstruyendo la observación de primer orden, pero a su tiempo, cuando se hayan podido minimizar las posibilidades de que la operación de observación de distinciones sea sólo una quimera. Esto significa que la observación de segundo orden no es sólo restrictiva de por sí, sino que debe intensificar metódicamente su carácter restrictivo. Estas reflexiones bloquean la posibilidad de identificar la observación de segundo orden con la distinción sistema/entorno, que está caracterizada por la simultaneidad (Luhmann 1990: 95 y ss.).

La deconstrucción se enlaza a la observación de latencias. Pero ¿dónde están las latencias? Habitualmente, las latencias se ocultan en los trozos de conversaciones 
más insospechados, en las miradas, en los olores, en los gestos de la cara, y en las estructuras de ordenamiento que no se dejan observar como tales, como ya expuso tempranamente Simmel (1908). A diferencia de la postura de Luhmann, nosotros pensamos, en aproximación a Simmel, que la latencia no tiene su origen en la imprenta y en la literatura, cuando el lector de novelas puede imaginarse los motivos de los personajes (Luhmann 1996: 1116-1117). La latencia nace de la oralidad (Ong 2006), de la gesticulación ininteligible desde fuera de las distinciones observables, del silencio, de la cerradura operativa de los sistemas de interacción conversacional, de la posibilidad de las inferencias indexicales (Albaladejo 2001).

\subsection{La observación de segundo orden como operación empírica y observable}

La observación de segundo orden debe ser una operación práctica, empírica, ejecutable y ejecutada en la sociedad. Si toda observación ocurre realmente y se produce operando con distinciones, entonces hay que indagar en las operaciones de comunicación de la sociedad, en los sistemas funcionales de la sociedad y en los sistemas de interacción de la sociedad. Por eso es que las teorías cognitivas pueden ser de gran utilidad, pero no reemplazan el imperativo de argumentar y describir una teoría de la observación que dé cuenta de la facticidad de la observación de primer orden y de las potencialidades de la observación de segunda orden, entendida como una 'metódica empírica' para las ciencias sociales. Nosotros pensamos que la supuesta paradoja distinción-indicación es una ficción. No se puede indicar algo que no se pueda distinguir de la otredad; lo que sucede es que la otredad es el unmaked state, es lo que el observador de primer orden no nos dice, sino que tenemos que desocultarlo. Esta es una condición de posibilidad para que se pueda ejecutar la observación de segundo orden, porque el trazado de la distinción, de acuerdo a las leyes de la forma, se realiza desde afuera hacia adentro, desde adentro hacia fuera se elimina la distinción (law of crossing) Por lo tanto, el lado que el observador de segundo orden debe marcar para "entrelazarse" con la observación de primer orden, es el unmaked state, y desde ahí tiene que cruzar el límite de la forma hacia adentro. Esto que parece evidente, es, sin embargo, no sólo dificultoso, sino además improbable.

\subsection{Las paradojas de la observación}

Las paradojas de la observación son dos, entre muchas (Luhmann 1993). Primero, que antes de la observación ya estuvo operando el observador, por lo que quien observe la observación y marque el unmarked state, marcará la otredad, el lado excluido -que el observador de primer orden no vio, y probablemente tampoco verá, es su punto ciego o la demostración de que no ve que no ve; o lo que el observador de primer orden pone-en-uso 'hace acccountable' sin necesidad de ir más allá. Esto convierte a la observación en paradójicamente reconstructiva, pero la observación de segundo orden puede re-temporalizar secuencialmente el 'cómo' de la observación, su contextualidad y su indexicalidad.

Segundo, la observación de segundo orden, al obligarse a entrelazarse a la observación de primer orden, pero también simplemente porque es una observación que obedece al manejo de distinciones, es también ciega, no ve que no ve. Esta es una paradoja que en realidad no tiene salida. En nuestra opinión, la posibilidad de desparadojización operando el observador por autología, es decir la autoimplicación reflexiva, arrastraría consigo la paradoja original. Como argumenta Luhmann: "Cuando se ensaya una reflexión tal, se castiga con una paradoja: lo distinguido es lo mismo. Y esto es válido, para repetirlo nuevamente, para el conocimiento y la acción y para el observador de primer orden como para el observador de segundo orden" (Luhmann 1997: 1134) 


\subsection{La distinción Esperar/Volver}

Si la observación de segundo orden es una actividad práctica, el observador debe poder observar su propio observar con la distinción esperar/volver. Antes describimos al sistema de observación en estado de espera, mediante el uso de la 'paciencia', algo similar a la "serenidad" [Gelassenheit] de Heidegger (1998) - más adelante vamos a explicar cómo se realiza la paciencia en la práctica. Lo vamos a formular de la siguiente manera: El observador debe poder observar la unidad de la diferencia esperar/volver, en su propio observar, 'esperando' hasta poder seguir el hilo de las conversaciones temporalizadas de los observadores de primer orden y 'volviendo' siempre a los registros originales, que son grabaciones, filmaciones, combinaciones video-audio o cualquier material 'reconstructor frío', 'desinteresado', 'indiferente' que le permita congelar la temporalización de lo-que-se-dijo (Ten Have 2004)

En otras palabras y esta vez siguiendo a Luhmann, el observador de segundo orden "tiene que observar cómo el observador observado maneja su paradoja; cómo resuelve su paradoja, cómo desparadojiza la paradoja de la observación" (Luhmann 1996: 75) La respuesta teórica a esta pregunta fundamental la entrega el mismo Luhmann con su "teorema de la doble contingencia" (Luhmann 1998: 113-139). La respuesta efectiva, real, práctica, se obtuvo haciendo de la observación de segundo orden una praxis investigativa. En efecto, la etnometodología de Garfinkel indica que los observadores-interactuantes de primer orden le dan una solución práctica a sus paradojas poniendo en uso la vaguedad, la ambigüedad inherente del lenguaje, dándose por satisfechos con explicaciones parciales que mantengan abiertas las expectativas recíprocas de la comunicación, y manteniendo un acuerdo mínimo entre ellos, pero sabiendo de lo que están hablando. (Garfinkel \& Sachs 1986).

Por lo tanto, hay que operar exactamente a la inversa de la recomendación subsiguiente de Luhmann. Jamás hay que abandonar la pregunta por el cómo, hay que permanecer indiferente respecto de qué, y rechazar categóricamente todas las interrogantes que inquieren sobre los por qué. Además, hay que evitar las reconstrucciones histórico-retrospectivas, $y$ en su lugar se debe cautelar estrictamente la temporalidad de lo-que-realmente-se-dijo, sin introducir las instrucciones 'críticas', propias del sabelotodo. Ahora podemos ofrecer otra salida a la segunda paradoja de la observación, a saber: la 'contingencia'. El reconocimiento explícito de que el mundo, o mejor dicho las comunicaciones de la sociedad, se ubican en el terrero de lo que no es necesario ni imposible. Por lo que todo podría ser diferente. Sin embargo, el argumento de la contingencia no puede ser una excusa vulgar para que el observador de segundo orden describa lo que se le ocurra como la observación de las observaciones ajenas.

\subsection{Las expectativas del observador de primer orden}

La observación de segundo orden debe incluir las expectativas, los intereses de los observadores de primer orden, los que tienen que haber sido registrados como intensivamente contextuales. Tienen que ser conversados, comunicados, debatidos, negociados y todo esto tiene que poderse mostrar con el material empírico de registro. Nosotros entendemos que tanto el principio 'No traces (todavía) ninguna distinción' así como la recomendación de 'saber esperar', como la instrucción de Spencer-Brown 'Stay where you are', deben imposibilitar que el observador de segundo orden trate de escapar de la indexicalidad de la comunicación de la sociedad, usando estrategias de sustitución y escogiendo el camino de la metodología, o simplemente 'sanando' la indexicalidad reemplazando lo-que-se-dijo, 
por conceptualizaciones sociológicas y confundiendo fatalmente las observaciones de primer orden con los 'grandes problemas de la sociología'.

\subsection{El posicionamiento del observador de segundo orden}

El observador de segundo orden, situado en el 'unmarked state' de la observación de primer orden, aunque sea incómodo y desagradable, tiene que buscar en las redes polifórmicas y contextuales de la comunicación de la sociedad, en lo que como forma de comunicar, se comunica/habla/conversa. Allí tiene que buscar el observador de segundo orden para obtener datos-valores que le permitan poder observar observaciones ajenas. Por lo tanto, la observación de segundo orden también debe ser una operación social de comunicación, pues si no lo fuera, permanecería encerrada en la clausura operativa del sistema psíquico del observador de segundo orden. Lo que no se comunica, no existe. El observador de segundo orden se enfrenta siempre al problema del tiempo, porque es el eterno atrasado. Por otro lado, debe enfrentar la temporalización propia de las comunicaciones de la sociedad, de los sistemas sociales y de los sistemas de interacción y la destemporalización de su propia observación respecto de la primera. El observador de segundo orden debe poder 'congelar' la temporalización de las conversaciones de los hablantes de primer orden, para lo cual debe usar recursos audiovisuales. ¿Por qué debe usar estos recursos y no las narraciones, las narraciones de narraciones o los libros, las revisiones bibliográficas o las novelas? La respuesta es simple: las observaciones de los observadores de primer orden, 'comunicadas' y 'conversadas', se ejecutan audiovisualmente.

Por último, la observación de segundo orden tiene 'cualidades tóxicas', porque desarma la posición del observador de primer orden que en cierto modo conservaba: infunde la sospecha en el mundo de la vida, sin que éste pueda abandonarse (Luhmann 2005: 162)

\section{Comentario final}

La observación de segundo orden no puede ni debe ser crítica, en otras palabras, "no se deja embaucar por la inherente imprecisión de la palabra krinein: separar, distinguir, juzgar, condenar" (Luhmann 2005: 168), lo que invalidaría la postura de Hellmann, quien cree constatar "muchos paralelos" entre la teoría crítica encarnada en la figura de Adorno y la teoría de sistemas de Luhmann (Hellmann 2002: 28). La opinión de Hellmann es equivocada e inaceptable, pues una cosa en inclinarse por un proyecto deconstructivo de sociología (Luhmann 2001a) y otra muy distinta es aproximarse al sectarismo elitista de Adorno, quién llegó al extremo de calificar de "castrados" a los amantes del jazz (Adorno 1971) y a distanciarse del movimiento estudiantil de 1968, llamando a los estudiantes esclavos de la industria cultural.RM

\section{Bibliografía}

Adorno, T. W. (1971). Zeitlose Mode. Zum Jazz. En R. Tiedermann (Ed), Adorno. Eine Auswahl (pp. 131-145). Frankfurt a.M.: Suhrkamp.

Albaladejo, T. (2001). Retórica, tecnología, receptores. Revista de Retórica y Teoría de la Comunicación, 1(1), 9-18.

An der Heiden, U. (1986). Ordnung und Chaos. Dialektik, 12, 154-167.

Baecker, D. (1993). Im Tunnel. En D. Baecker (Ed.), Kalkül der Form (pp. 12-37). Frankfurt a.M.: Suhrkamp.

Baecker, D. (Ed.) (1993a). Probleme der Form. Frankfurt a.M.: Suhrkamp. 
Corsi, G. (1993a). Die dunkle Seite der Karriere. En D. Baecker (Ed.), Probleme der Form (pp. 252-265). Frankfurt a.M.: Suhrkamp.

Derrida, J. (1972). Die Schrift und die Differenz. Frankfurt a.M.: Suhrkamp.

Eckert, P. (2008). Variation and the indexical Field. Journal of Sociolinguistic, 12(4), 453-476.

Esposito, E. (1993). Ein zweiwertiger nicht-selbständiger Kalkül. En D. Baecker (Ed.), Kalkül der Form (pp. 96-1 11). Frankfurt a.M.: Suhrkamp.

Flores, R. (2009). Observando observadores. Una introducción a las técnicas cualitativas de investigación social. Santiago de Chile: Ediciones Universidad Católica de Chile.

Fontdevila, J., Opazo, P. \& White, H. (2011). Order at the Edge of Chaos: Meaning from Netdom Switchings across Functional Systems. Sociological Theory, 29(3), 179198.

Garfinkel, H. \& Sachs, H. (1986). On formal Structures of Practical Actions. En Harold Garfinkel (Ed.), Ethnomethodological Studies of Work (pp. 157-190). London/New York: Routledge.

Gödel, K. (1940). The Consistency of the Continuum Hypothesis. New York: Princeton University Press.

Gougen, J. \& Varela, F. (1979). Systems and distinctions: Duality and Complementarity. International Journal of General Systems, 5(1), 31-43.

Günther, G. (1976). Beiträge zur Grundlegung einer operationsfähigen Dialektik, Band 1. Hamburg: Felix Meiner Verlag.

Günther, G. (2002). Erkennen und Wollen. Ein Beitrag zur kybernetischen Theorie der Subjektivität. En, Das Bewusstsein der Maschinen. Eine Metaphysik der Kybernetik (pp. 229-285). Baden-Baden: Agis Verlag.

Hellmann, K. U. (2002). Aristoteles und wir. En K. U. Hellmann \& R. Schmalz -Bruns (Eds.), Theorie der Politik. Niklas Luhmann politische Soziologie (pp. 10-39). Frankfurt a.M.: Suhrkamp.

Heidegger, M. (1998). Filosofía, ciencia y técnica. Santiago de Chile: Editorial Universitaria.

Husserl, E. (1980). Ideen zu einer reinen Phänomenologie und Phänomenologischer Philosophie. Tübingen: Niemayer.

Husserl, E. (1991). La crisis de las ciencias europeas y la fenomenología trascendental. Barcelona: Crítica.

Hutter, M. (1993a). Die frühe Form der Münze. En D. Baecker (Ed.), Probleme der Form (pp. 159-179). Frankfurt a.M.: Suhrkamp.

Kaehr, R. (1993). Disseminatorik: Die Logik der "Second Order Cybernetics". Von den Gesetzen der Form zur Logik der Reflexionsform. En D. Baecker (Ed.), Kalkül der Form (pp. 152-197). Frankfurt a.M.: Suhrkamp.

Kelly-Bootle, S. (1990). Logic Is Not Enough. Computer Language, 7, 135-139.

Lacan, J. (1973). Die Ausrichtung der Kur und die Prinzipien ihrer Macht. En J. Lacan, Schriften. Frankfurt a.M.: Suhrkamp.

Luhmann, N. (1990a), Sthenographie. En N. Luhmann et al. (Eds.), Beobachter. Konvergenz der Erkenntnistheorie? (pp. 119-138). München: Fink Verlag.

Luhmann, N. (1992). Kontingenz als Eigenwert der modernen Gesellschaft. En N. Luhmann, Beobachtungen der Moderne. Opladen: Westdeutscher Verlag.

Luhmann, N. (1993). Die Paradoxie der Form. En D. Baecker (Ed.), Kalkül der Form (pp. 197-212). Frankfurt a.M.: Suhrkamp.

Luhmann, N. (1996). La ciencia de la sociedad. México D.F.: Anthropos, UIA.

Luhmann, N. (1997). Die Gesellschaft der Gesellschaft. Frankfurt a.M.: Suhrkamp.

Luhmann, N. (1998): El conocimiento como construcción. En N. Luhmann, Teoría de los sistemas sociales II (pp. 67-90). Talcahuano: UIA, Universidad de Los Lagos.

Luhmann, N. (1998). Sistemas Sociales. Lineamientos para una teoría general. México D.F.: Anthropos, Universidad Iberoamericana, Pontificia Universidad Javeriana. 
Luhmann, N. (2001). Autopoiesis als soziologischer Begriff. En O. Jahrhaus (Ed.), Niklas Luhmann. Aufsätze und Reden (pp. 137-158). Stuttgart: Reclam.

Luhmann, N. (2001a). Dekonstruktion als Beobachtung zweiter Ordnung. En 0. Jahrhaus (Ed.), Niklas Luhmann. Aufsätze und Reden (pp. 262-296). Stuttgart: Reclam.

Luhmann, N. (2005), El arte de la sociedad. México D.F.: Herder, UIA.

Luhmann, N. (1990). Gleichzetigkeit und Synchonisation. En N. Luhmann, Soziologische Aufklärung 5 (pp. 95-130). Opladen: Westdeutscher Verlag.

Maturana, H. (1998). La objetividad. Un argumento para obligar. Santiago de Chile: Dolmen.

Maturana, H. \& Varela, F. (1984). El árbol del conocimiento. Santiago de Chile: Editorial Universitaria.

Ong, W. (2006). Oralidad y Escritura. Tecnologías de la palabra. Buenos Aires: FCE.

Robles, F. (2002). Sistemas de interacción, doble contingencia y autopoiesis indexical. Cinta de Moebio, 15.

Russell, B. (1972). Lancelot Law Whyte, Review of the Laws of Form. British Journal of the Philosophy of Science, 23(3), 291-292.

Simmel, G. (1908). Soziologie. Untersuchungen über die Formen der Vergesellschaftung. Berlin: Duncker \& Humblot.

Simon, F. (1993). Mathematik und Erkenntnis: Eine Möglichkeit, die 'Laws of Form' zu lessen. En D. Baecker (Ed.), Kalkül der Form (pp. 38-57). Frankfurt a.M.: Suhrkamp.

Simon, F. (1993a). Die andere Seite der Krankheit. En D. Baecker (Ed.), Probleme der Form (pp. 266-289). Frankfurt a.M.: Suhrkamp.

Spencer-Brown, G. (1997). Gesetze der Form. Lübeck: Bohmeier.

Stichweh, R. (1993a). Die Form der Universität. En D. Baecker (Ed.), Probleme der Form (pp. 181-211). Frankfurt a.M.: Suhrkamp.

Ten Have, P. (2004). Understanding Qualitative Research and Ethnomethodology. London: Sage.

Torres Nafarrate, J. (1999). In memoriam. El legado sociológico de Niklas Luhmann. Sociológica, 14 (40), 89-108.

Varga, M \& Matzka. R (1993). Motive und Grundgedanken der „Gesetze der Form“ . En D. Baeker (Ed.), Kalkül der Form (pp. 58- 85), Frankfurt a.M.: Suhrkamp.

Von Foerster, H. (1993). Die Gesetze der Form. En D. Baecker (Ed.), Kalkül der Form (pp. 9-11). Frankfurt a.M.: Suhrkamp.

Von Foerster, H. (1996). Cibernética de la cibernética. En H. von Foerster, Las semillas de la cibernética (pp. 89-93). Barcelona: Gedisa.

Von Foerster, H. (1998). Wahrheit ist die Erfindung eines Lügners. Heidelberg: Carl Auer Systeme Verlag.

Von Foerster, H. (2003). Cybernetics and Circularity. The Cybernetics Society, noviembre.

Von Foerster, H. (1990). Construyendo una realidad. En P. Watzlawick (Ed.), La realidad inventada. ¿Cómo sabemos los que creemos saber? (pp. 38-56). Barcelona: Gedisa. ,

Winter, W. (1999). Theorie des Beobachters. Skizzen zur Architektonik eines Metatheoriesystems. Frankfurt.a.M.: Neue Wissenschaft.

Wittgenstein, L. (1957). Tactactus Logico-Philosophicus. Madrid: Revista de Occidente Editorial.

Sobre el autor

Fernando Robles es actualmente Profesor Asociado del Departamento de Sociología y Antropología de la Universidad de Concepción, Chile. Doctor en sociología por la Universidad Ludwig-Maximilian de Munich, Alemania. Entre sus áreas de especialización se encuentran: riesgo social, sociología política, teoría de sistemas sociales, desigualdad y exclusión social, etnometodología y análisis conversacional. 
Entre sus últimas publicaciones se destacan: El análisis conversacional desde la etnometodología. Un manual de trabajo (2011), Hablo contigo si tú hablas conmigo: metódica y análisis de los sistemas de interacción (Escaparate: 2006).

hrobles@udec.cl

\section{Contacto}

Departamento de Sociología y Antropología

Facultad de Ciencias Sociales, $4^{\circ}$ Piso

Casilla 160-C

Barrio Universitario

Universidad de Concepción

Chile.

Recibido: Junio 2012

Aceptado: Agosto 2012 